\title{
Amplified X-Ray Emission from Core-Ionized Diatomic Molecules
}

\author{
Victor Kimberg and Nina Rohringer \\ Max Planck Institute for the Physics of Complex Systems, 01187 Dresden, Germany \\ and Max Planck Advanced Study Group, CFEL, Luruper Chaussee 149, 22761 Hamburg, Germany
}

(Received 24 August 2012; published 22 January 2013)

\begin{abstract}
We predict high-gain $\mathrm{x}$-ray lasing in molecular nitrogen by ultrafast core ionization with an $\mathrm{x}$-ray freeelectron laser source. To estimate the spectral and temporal output of this molecular x-ray laser, we solve generalized Maxwell-Bloch equations, keeping track of the electronic and nuclear degrees of freedom. The spectrum of the amplified x-ray emission shows a strong dependence on the gain-length product. Whereas the emission at small gain length is similar to the relatively broad x-ray fluorescence band, the spectrum is determined by a single frequency in the linear gain region. The vibrational wave packet dynamics during the $\mathrm{x}$-ray emission process is examined. By preparation of the initial vibrational quantum state, the $\mathrm{x}$-ray emission frequency can be tuned within the fluorescence band. The present scheme is applicable to other homo- and heteronuclear diatomic systems, thereby extending the spectral range of coherent $\mathrm{x}$-ray radiation sources based on amplification on bound transitions.
\end{abstract}

X-ray free-electron lasers (XFELs) [1-3] open the pathway to study quantum optics [4-8] and quantum electronics [9-11] in the $\mathrm{x}$-ray regime. A prominent prospective application of XFELs is nonlinear spectroscopy in the x-ray region, to study the dynamics and correlations in many-body quantum systems: Whereas the very ambitious goal of x-ray four-wave mixing $[12,13]$ and multidimensional $\mathrm{x}$-ray spectroscopy [14] would require a sequence of several well controlled $x$-ray pulses of preferably sub-fs duration and distinct photon energy, other schemes are less demanding in terms of x-ray pulse properties. All x-ray pump-probe schemes such as impulsive Raman scattering [14-16] or stimulated inelastic x-ray scattering [17] require two fs or sub-fs $x$-ray pulses of preferentially distinct photon energy, separated by a welldefined delay time. Although recent progress in selfseeding schemes in the hard $\mathrm{x}$-ray regime $[18,19]$ gives a positive outlook on a better control of the longitudinal coherence and spectral properties of XFELs, seeded twocolor schemes might be difficult to realize in the near future [19]. One possible solution to produce two-color $\mathrm{x}$-ray sources was recently demonstrated: By the focusing of an XFEL into a dense gas target, a secondary x-ray beam was generated by nearly saturated amplification of $\mathrm{X}$-ray fluorescence in neon $[9,20]$. The population inversion in this atomic x-ray laser was achieved by ultrafast inner-shell photoionization [21] by the XFEL beam, tuned above the $K$ edge of the gain medium.

Here, we propose an extension of this method to diatomic molecules, thereby extending the accessible photon-energy range from a quite limited number of atomic gas targets to a larger variety of molecular targets. Due to the additional nuclear degree of freedom, $x$-ray lasing in molecules offers new opportunities. A discrete tunability of the emission of the atomic constituent $A$ can be reached by changing the second atomic constituent $B$, which generally results in an energy shift of the $K$-shell transition in constituent $A$. Moreover, we suggest the possibility of fine-tuning of the transition by controlling the vibrational initial state. The influence of vibrational dynamics on the x-ray amplification process is crucial: Depending on the nuclear dynamics, three different lasing scenarios can be discerned, as sketched in Fig. 1. Shown are the electronic potential surfaces of three different possible schemes. The impinging XFEL pump beam prepares the molecules in an electronically core-excited state, by either resonant excitation or photoionization. This intermediary state is either a molecular bound state, or dissociative. In the case of a dissociative core-excited state [Fig. 1(a)], radiative decay can either happen in the molecule (slowly dissociative state) or in the atom, if dissociation is fast in comparison to the lifetime of the upper state [22-24]. In the first case, a spectrally broad emission feature is expected. Since the gain cross section typically scales as the inverse bandwidth of the emission, this would result in small gain. This is also expected in the case of a core-excited bound state coupled to a dissociative final state [Fig. 1(b)]. Here, we discuss lasing between molecular bound states [Fig. 1(c)] and present calculations based on a generalized MaxwellBloch approach for $\mathrm{N}_{2}$. We treat both electronic and vibrational degrees of freedom, thereby revealing the importance of wave packet dynamics in stimulated $\mathrm{x}$-ray processes.

By tuning a focused XFEL beam above the $K$ edge of nitrogen $(410 \mathrm{eV})$, a core hole in $\mathrm{N}_{2}$ is produced by $K$-shell ionization. This core-excited intermediate state has a short lifetime of $\approx 6.6 \mathrm{fs}$ [25], dominated by the Auger decay. If core-ionization rates are comparable to the Auger rate, a sizeable population inversion in molecular nitrogen can be achieved. Despite the small fluorescence yield in nitrogen $\left(\sim 5 \times 10^{-3}[26]\right)$, spontaneously emitted $\mathrm{x}$-ray photons 
(a)

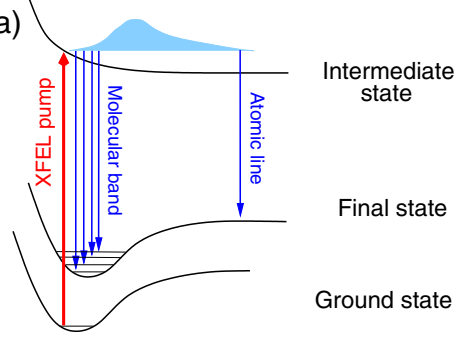

(b)

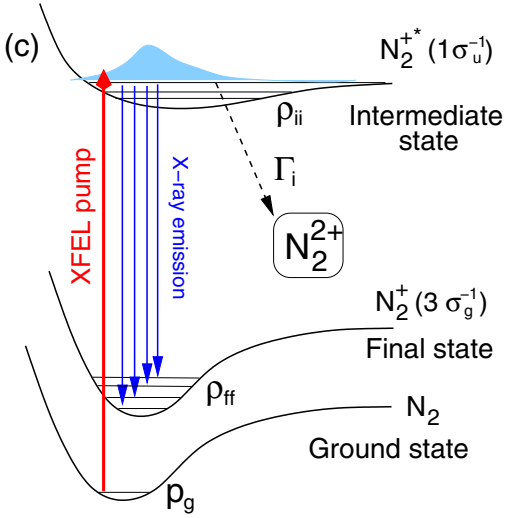

FIG. 1 (color online). Lasing schemes in diatomic molecules. (a) Dissociative intermediate state: radiative decay in the molecule or in the atom (a), dissociative final state (b), bound-bound lasing (c). Here, $p_{g}, \rho_{i i}$, and $\rho_{f f}$ are the populations of the vibrational sublevels of the ground, core-ionized, and final electronic states, respectively; $\Gamma_{i}$ is the inverse lifetime of the core-ionized state.

can get exponentially amplified, similarly to the atomic case $[9,20]$. The geometry of this single-pass $x$-ray amplifier is determined by the focus properties of the pump source. Typically, a long $(5-10 \mathrm{~mm})$, narrow $(1 \mu \mathrm{m})$ focus of the XFEL creates an elongated plasma channel of coreinverted molecules. Fluorescence photons emitted at the entrance of the plasma channel stimulate radiative emission in direction of the propagating pump pulse and get exponentially amplified. The level scheme of the lasing transition is schematically shown in Fig. 1(c). We assume that nitrogen is initially in the ground state $\left({ }^{1} \Sigma_{g}^{+}\right)$. Core ionization promotes the molecule to the almost degenerate bound states $1 \sigma_{g}^{-1}$ and $1 \sigma_{u}^{-1}$. Radiative decay of these states proceeds mainly to the bound final states $2 \sigma_{u}^{-1}$, $1 \pi_{u}^{-1}$, and $3 \sigma_{g}^{-1}$ [27]. The three transitions are well separated in energy on the scale of the typical vibrational broadening. Since the transition to the $3 \sigma_{g}^{-1}$ state is the strongest, we restrict our analysis to the lasing on the $1 \sigma_{u}^{-1} \rightarrow 3 \sigma_{g}^{-1}$ transition $(\approx 395 \mathrm{eV})$, which shows the highest gain.

To model the amplified $\mathrm{x}$-ray emission (AXE), we use a generalized Maxwell-Bloch approach. By employment of the paraxial wave approximation and the assumption of a slowly varying envelope of the electric field [28], the propagation and amplification of the electric field associated with the AXE is modeled by

$$
\frac{\partial E}{\partial z}+\frac{1}{c} \frac{\partial E}{\partial t}=-\imath \frac{2 \pi N}{c \omega_{L}} \sum_{i, f} \omega_{i f}^{2} \tilde{\rho}_{f i} d_{i f} e^{l\left(\omega_{i f}-\omega_{L}\right) t}
$$

where $E(t, z)$ is the slow varying complex component of the AXE electric field, $c$ is the speed of light in vacuum, $N$ is the molecular density, $\omega_{L}$ is the x-ray emission frequency, and $\omega_{i f}$ is the transition frequency between vibrational sublevels $i$ and $f$ of the intermediate and final electronic states respectively. With the use of the FranckCondon principle, the transition matrix element $d_{i f}=$ $d_{e}\left\langle\nu_{i} \mid \nu_{f}\right\rangle$ is represented as a product of the electronic dipole moment $d_{e}=0.07$ a.u. computed on the HartreeFock level using the GSCF3 code [29] and assumed to be independent of the internuclear distance and the overlap integral of the vibrational wave functions $\left|\nu_{j}\right\rangle(j=i, f)$. The vibrational energies and wave functions are calculated by using the potential-energy curves from Ref. [27]. The right-hand side of Eq. (1) involves the polarization change of the medium, determined by the off-diagonal matrix element of the reduced density matrix of the cation $\tilde{\rho}_{f i}$. The evolution of the reduced ionic density matrix is governed by the generalized Bloch equations

$$
\begin{aligned}
\dot{\rho}_{i f}(\theta ; z, t)= & -\frac{\Gamma_{i}}{2} \rho_{i f}+\imath \sum_{f^{\prime}} \rho_{f^{\prime} f} R_{i f^{\prime}}^{*}-\imath \sum_{i^{\prime}} \rho_{i i^{\prime}} R_{i^{\prime} f}^{*} \\
& +\left(\rho_{i i}-\rho_{f f}\right) S(t, z), \\
\dot{\rho}_{i i^{\prime}}(\theta ; z, t)= & -\Gamma_{i} \rho_{i i^{\prime}}+P_{i i^{\prime}}+i \sum_{f}\left(\rho_{f i^{\prime}} R_{i f}^{*}-\rho_{i f} R_{i^{\prime} f}\right), \\
\dot{\rho}_{f f^{\prime}}(\theta ; z, t)= & i \sum_{i}\left(\rho_{i f^{\prime}} R_{i f}-\rho_{f i} R_{i f^{\prime}}^{*}\right),
\end{aligned}
$$

where $R_{i f}(z, t)=E(z, t) d_{i f} \cos \theta e^{i\left(\omega_{L}-\omega_{i f}\right) t}$ are the complex Rabi frequencies and $\theta$ is the angle between the electric field polarization and the direction of the electronic transition dipole moment. We do not account for the rotational motion, since rotational periods are long compared to the relevant time scale of the lasing process. The angle $\theta$ is treated as a parameter. To perform averaging over the orientation of the dipole moment, we solve Eqs. (2) independently for a number of angles $\theta_{j}$, assuming a uniform distribution of aligned molecules in a cone $\left[0, \ldots, \theta_{j_{\max }}\right]\left(\theta_{j_{\max }}=\pi\right.$ for an ensemble of unaligned molecules). Then, the averaged polarization $\tilde{\rho}_{i f}(z, t)=$ $\sum_{j=1}^{j_{\max }} \rho_{i f}\left(\theta_{j} ; z, t\right) / j_{\max }$ is used in Eq. (1). The Auger decay of the intermediate states $\left(\Gamma_{i}=0.1 \mathrm{eV}\right)$ is taken into account phenomenologically in Eq. (2). The pump rate of the intermediate electronic state $P_{i i^{\prime}}$ is determined by

$$
\begin{aligned}
P_{i i^{\prime}}(z, t) & =p_{g}(z, t) e^{i\left(\epsilon_{i}-\epsilon_{i^{\prime}}\right) t}\left\langle\nu_{g} \mid \nu_{i}\right\rangle\left\langle\nu_{i^{\prime}} \mid \nu_{g}\right\rangle \Phi_{p}(z, t) \sigma_{1 \sigma_{u}}, \\
p_{g}(z, t) & =e^{-\sigma_{\text {ion }} \int_{0}^{t} \Phi_{p}\left(z, t^{\prime}\right) d t^{\prime}},
\end{aligned}
$$

where $p_{g}(z, t)$ is the ground-state population and

$$
\Phi_{p}(z, t)=\Phi_{p}(z=0, t) e^{-p_{g}(z, t) N z \sigma_{\text {ion }}}
$$


is the flux of the XFEL pulse. Owing to the dipole selection rules, the final state can be only reached via the ungerade intermediate core-hole state $1 \sigma_{u}^{-1}$. This is taken into account in Eq. (3): the cross section of the active channel $\sigma_{1 \sigma_{u}}$ is half the total ionization cross section $\sigma_{\text {ion }}$ [25,27]. The results of our calculations are averaged numerically over the stochastic polarization source term $S(z, t)$ in Eq. (2) $[28,30]$. In what follows [except Fig. 2(a)], we suppose a partially aligned ensemble of molecules with $\left\langle\cos ^{2} \theta\right\rangle=0.8$.

Our numerical results show that $\mathrm{x}$-ray lasing in diatomic molecules should be achievable at presently available XFEL sources. We assumed a Gaussian temporal profile of the XFEL pulse, a good approximation in the case of nonresonant excitation, where the actual spectrum of the XFEL pulse does not matter. We chose parameters typically achievable at present-day XFEL sources: a pulse duration of $50 \mathrm{fs}, 3.6 \times 10^{12}$ photons per pulse, and a focus radius of $1.5 \mu \mathrm{m}$. Figure 2(a) shows the average number of photons in the AXE pulse as a function of the propagation length, assuming a molecular density $N=$ $2.5 \times 10^{19} \mathrm{~cm}^{-3}$. The density is matched to the interaction length and the gain cross section so that the integrated gain-length product is $\approx 20$, i.e., the typical value for

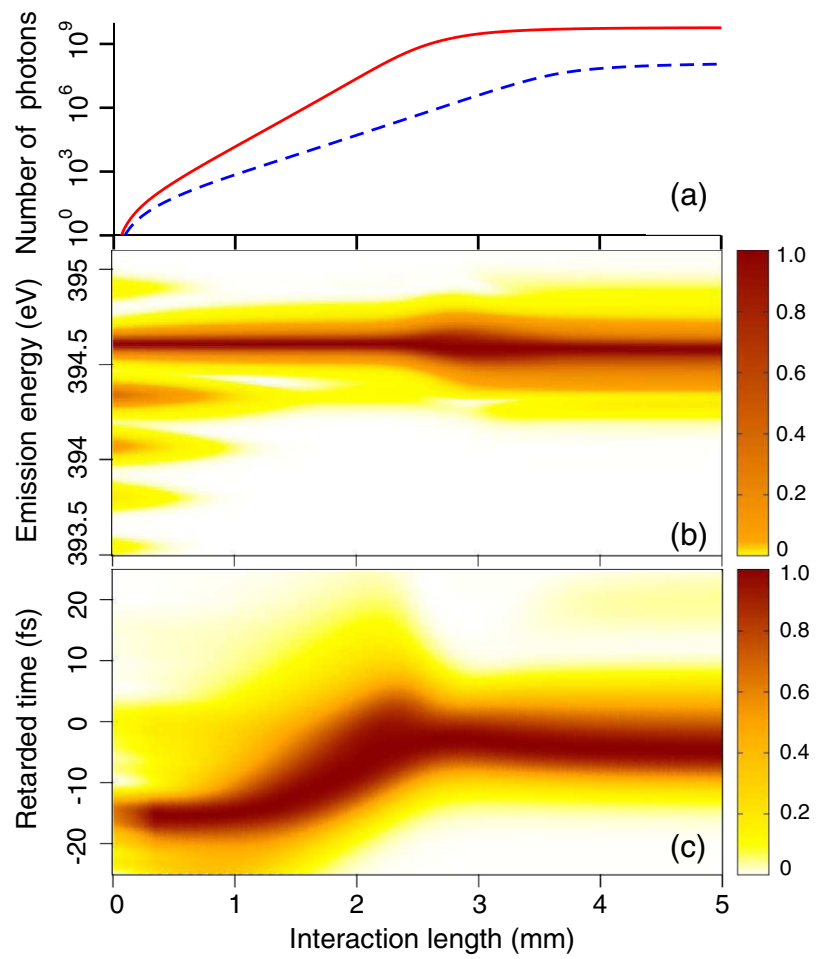

FIG. 2 (color online). (a) Number of photons, (b) spectrum, and (c) normalized temporal pulse profile (in the frame $t^{\prime}=$ $t-z / c$ of the propagating XFEL pulse) of the AXE pulse as a function of the interaction length $z$. In (a), we also plot the number of AXE photons for an ensemble of unaligned molecules (blue dashed line). The spectrum (b) is computed as a double Fourier transform of the $E$-field correlation function. reaching gain saturation [9]. Before reaching saturation at a length of $\approx 2.5 \mathrm{~mm}$, the number of photons in the AXE line grows exponentially with a gain of $72 \mathrm{~cm}^{-1}$. At saturation, the rate of stimulated emission equals the Auger decay rate and the gain reduces, resulting in a bending of the gain curve. At the end of the medium, the AXE pulse contains $\approx 6 \times 10^{9}$ photons, corresponding to a conversion efficiency of $\approx 2 \times 10^{-3}$. The integrated gain-length product for an ensemble of unaligned molecules [see dashed line in Fig. 2(a)] is $\approx 14$. Partial alignment of the molecular gain medium is therefore important to achieve gain saturation.

Figure 2(b) shows the evolution of the emission spectrum as a function of penetration depth. Below the onset of the linear gain region $(z \ll 1 \mathrm{~mm})$, the AXE spectrum consists of several emission lines of transitions between vibrational sublevels, similar to the $\mathrm{x}$-ray fluorescence spectrum [27]. In the linear gain region, a single transition energy $\left(\omega_{00}=394.61 \mathrm{eV}\right)$ picks up, suppressing the amplification of the other vibrational channels, so that at $z \approx 1.5 \mathrm{~mm}$, the spectrum transforms into a narrow line. The width of the line is determined by the natural lifetime broadening of the core-excited state $\Gamma_{i}$. At saturation $(z \gtrsim$ $2.5 \mathrm{~mm}$ ), the AXE line rebroadens (saturation broadening) and the line shape is determined by nonlinear effects. Deep in saturation $(z \gtrsim 4 \mathrm{~mm})$, the emission shifts towards lower energy and develops an asymmetric line shape [see panel $\nu_{g}=0$ in Fig. 4(b)]. Note that the shifted emitted frequency does not correspond to a molecular transition. However, the emitted frequency corresponds to the fourwave mixing frequency $\omega=2 \omega_{00}-\omega_{22}$ of the vibrational channels $\omega_{i f}=\omega_{00}$ and $\omega_{22}$ [11]. It is astonishing that the relatively weak four-wave mixing process results in a shift of the line. The induced shift, however, is on the scale of the Rabi frequency (at $z=3 \mathrm{~mm}$, the averaged Rabi frequency is $\left\langle\left|R_{00}\right|\right\rangle \approx 0.03 \mathrm{eV}$ ). This implies that the nonlinear four-wave mixing process is seeded by the saturation broadened $\mathrm{x}$-ray emission, resulting in a shift of the emission energy and an asymmetric line profile.

Figure 2(c) shows the evolution of the temporal profile of the emitted AXE. In the linear gain region, the peak maximum of the emitted AXE pulse shifts towards later times in the frame propagating with the XFEL pulse. The AXE pulse propagating on a resonant high-gain transition experiences a slowdown [31]. Strong absorption of the XFEL pulse at the leading edge of the pulse results in a shift of the XFEL pulse front so that the intermediate-state population [shown in Fig. 3(a) for three positions in the medium] and the population inversion shift to later times and temporally overlap with the AXE pulse maximum at every position $z$. This effect of gain guiding is crucial for the efficiency of the amplification process [22]. Omitting the XFEL absorption (not shown here) counterintuitively results in considerably smaller gain, since the AXE pulse trains behind the population inversion. 


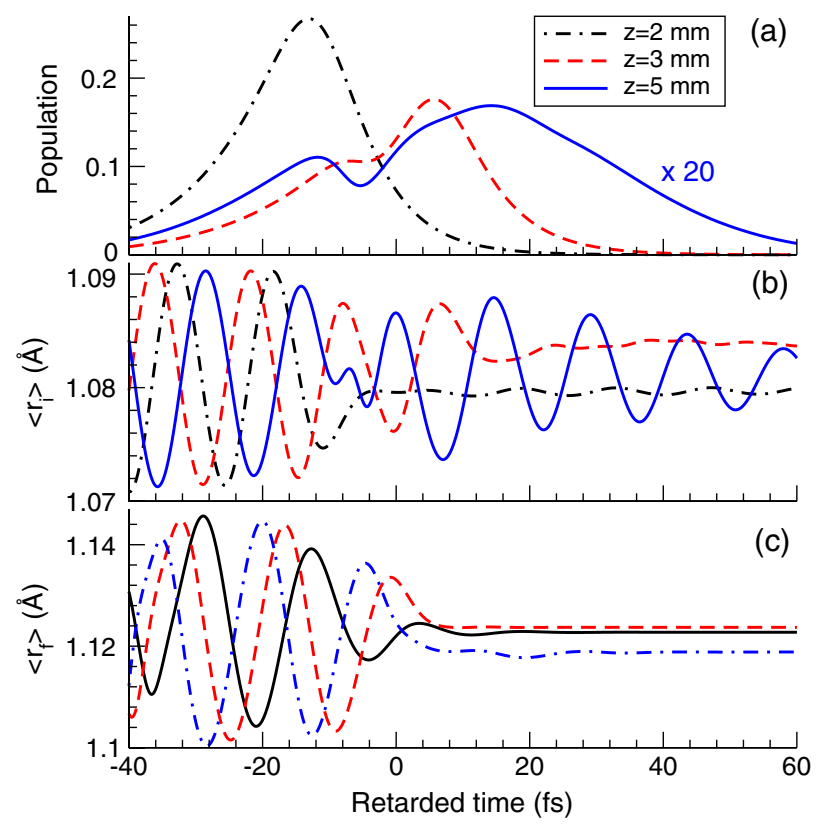

FIG. 3 (color online). Temporal dependence of the total population of the core-ionized state $\sum \rho_{i i}$ (a) and the expectation value of the internuclear distance of the intermediate $\left\langle r_{i}\right\rangle$ (b) and final $\left\langle r_{f}\right\rangle$ (c) state vibrational wave packet at $z=2,3$, and $5 \mathrm{~mm}$.

The strongly driven molecular system shows interesting vibrational wave packet dynamics. As in the optical case [32], this dynamics will affect the emission properties of the molecule [33]. In Fig. 3, we show the expectation value of the internuclear distance of the core-excited [Fig. 3(b)] and final state wave packets [Fig. 3(c)] for three different positions in the gain medium, characterized by increasing AXE intensity. Initially, the intermediate state wave packet oscillates with a period $\approx 15$ fs. Along with the decay of the occupation probability of the intermediate state, shown in Fig. 3(a), these oscillations are damped. At $z=5 \mathrm{~mm}$, the internuclear distance in the intermediate wave packet shows a jump at $t^{\prime}=-8 \mathrm{fs}$, along with a dip in the population of the intermediate state. This is a signature of Rabi flopping mediated by the saturated AXE field, since rapid passage to the final state and back to the core-excited state goes along with a change in the internuclear distance. In the final state wave packet [Fig. 3(c)], a shift of the final bond length is observed with increasing AXE intensity. After the decline of the x-ray pulses, no oscillations in the bond length are seen, implying incoherence of the final vibrational wave packet. The final states occupation happens over a time of $\approx 50 \mathrm{fs}$ (given by the duration of the population of the intermediated state), which is greater than the typical vibrational period ( $\approx 15 \mathrm{fs})$. Evidently, the vibrational final states populated at different times are out of phase; i.e., a loss of coherence in the final state vibrational wave packet has occurred. For $\mathrm{N}_{2}$, the field-induced nuclear dynamics is rather weak, due to the similarity between the intermediate and final

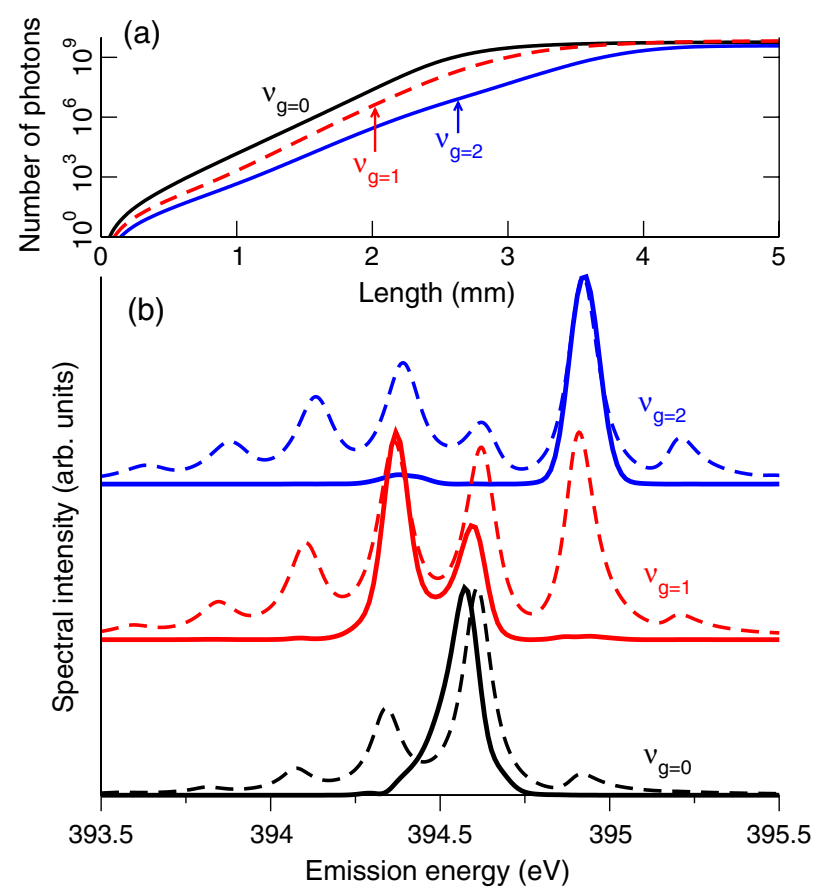

FIG. 4 (color online). (a) Number of AXE photons and (b) AXE (solid) and fluorescence spectra (dashed) computed for different vibrational initial states $\nu_{g}, g=0,1,2$.

potential energy curves. More pronounced effects are expected in the general case, especially when dissociative states are involved in the scattering process. Hence, in addition to electron dynamics, vibrational dynamics can play an eminent role in ultrafast $\mathrm{x}$-ray stimulated processes.

In general, the fluorescence spectrum depends on the nuclear wave packets of the ground and intermediate electronic states $[34,35]$. Thus, by preparation of the initial state in a specific vibrational state, for example, by IR Raman excitation, the emission frequency of the AXE can be tuned within the fluorescence band. To demonstrate this effect, we run simulations for different vibrational initial states $\left(\nu_{g}=0,1,2\right)$. Due to a decrease of the Franck-Condon factors, the gain for initial vibrational levels $\nu_{g}=1,2$ is smaller than that for $\nu_{g}=0$ [see Fig. 4(a)]. The respective spectra are shown in Fig. 4(b). Evidently, the emitted AXE frequency coincides with the strongest peak of the fluorescence signal and can be finetuned by addressing specific vibrational levels. It is worth noting that any initial vibrational wave packet which differs sufficiently from the vibrational ground state would result in a change of the AXE spectrum.

In conclusion, on the basis of our theoretical estimate, $\mathrm{x}$-ray lasing from diatomic molecules pumped by highintensity $\mathrm{x}$-ray pulses seems realizable at presently available XFEL sources. A feasibility study for $\mathrm{N}_{2}$ was presented. Unlike the broad $\mathrm{X}$-ray fluorescence band, the amplified $\mathrm{x}$-ray emission is emitted at a single vibrational transition. The resulting transform-limited $\mathrm{x}$-ray pulses 
have an extremely narrow linewidth $(0.2 \mathrm{eV})$ and short pulse duration (15 fs). The emission frequency can be tuned within the $\mathrm{x}$-ray fluorescence band by manipulating the vibrational wave packet in the electronic ground state. Furthermore, the molecular gain medium offers the possibility of polarization control of the emitted $\mathrm{x}$-ray radiation by controlling the molecular orientation and thereby the orientation of the transition dipole moment. Although this case study focuses on $\mathrm{N}_{2}$, the scheme can be applied to other homo- and heteronuclear diatomic systems, thereby extending the spectral range of this molecular-based $\mathrm{x}$-ray lasing scheme. Fine tunability of the molecular laser line in conjunction with the tunable XFEL radiation results in a universal two-color $\mathrm{x}$-ray radiation source, enabling advanced all-x-ray pump-probe experiments.

We thank Oriol Vendrell for fruitful discussions.

[1] P. Emma et al., Nat. Photonics 4, 641 (2010).

[2] W. Ackermann et al., Nat. Photonics 1, 336 (2007).

[3] T. Ishikawa et al., Nat. Photonics 6, 540 (2012).

[4] R. Röhlsberger, K. Schlage, B. Sahoo, S. Couet, and R. Rüffer, Science 328, 1248 (2010).

[5] R. Röhlsberger, H.-C. Wille, K. Schlage, and B. Sahoo, Nature (London) 482, 199 (2012).

[6] S. Shwartz, R. N. Coffee, J. M. Feldkamp, Y. Feng, J. B. Hastings, G. Y. Yin, and S. E. Harris, Phys. Rev. Lett. 109, 013602 (2012).

[7] M. Nagasono, J. R. Harries, H. Iwayama, T. Togashi, K. Tono, M. Yabashi, Y. Senba, H. Ohashi, T. Ishikawa, and E. Shigemasa, Phys. Rev. Lett. 107, 193603 (2011).

[8] Nonlinear Optics, Quantum Optics, and Ultrafast Phenomena with X-Rays, edited by B. Adams (Kluwer Academic Publishers, Dordrecht, The Netherlands, 2003).

[9] N. Rohringer et al., Nature (London) 481, 488 (2012).

[10] E. Kanter et al., Phys. Rev. Lett. 107, 233001 (2011).

[11] Y.-P. Sun, J.-C. Liu, C.-K. Wang, and F. Gel'mukhanov, Phys. Rev. A 81, 013812 (2010).

[12] S. Tanaka and S. Mukamel, Phys. Rev. Lett. 89, 043001 (2002).
[13] S. Mukamel, Phys. Rev. B 72, 235110 (2005).

[14] J.D. Biggs, Y. Zhang, D. Healion, and S. Mukamel, J. Chem. Phys. 136, 174117 (2012).

[15] I. V. Schweigert and S. Mukamel, Phys. Rev. A 76, 012504 (2007).

[16] D. Healion, H. Wang, and S. Mukamel, J. Chem. Phys. 134, 124101 (2011).

[17] B. D. Patterson, SLAC Technical Note SLAC-TN-10-026, 2010.

[18] J. Amann et al., Nat. Photonics 6, 693 (2012).

[19] G. Geloni, V. Kocharyan, and E. Saldin, J. Mod. Opt. 58, 1391 (2011).

[20] N. Rohringer and R. London, Phys. Rev. A 80, 013809 (2009).

[21] M. A. Duguay and G. P. Rentzepis, Appl. Phys. Lett. 10, 350 (1967).

[22] Q. Miao, J.-C. Liu, H. Ågren, J.-E. Rubensson, and F. Gel'mukhanov, Phys. Rev. Lett. 109, 233905 (2012).

[23] P. Morin and I. Nenner, Phys. Rev. Lett. 56, 1913 (1986).

[24] P. Morin and C. Miron, J. Electron Spectrosc. Relat. Phenom. 185, 259 (2012).

[25] B. Kempgens, A. Kivimäki, M. Neeb, H. M. Köppet, A. M. Bradshaw, and J. Feldhaus, J. Phys. B 29, 5389 (1996).

[26] J. H. Hubbell, P. N. Trehan, N. Singh, B. Chand, D. Mehta, M. L. Garg, R. R. Garg, S. Singh, and S. Puri, J. Phys. Chem. Ref. Data 23, 339 (1994).

[27] P. Glans, P. Skytt, K. Gunnelin, J.-H. Guo, and J. Nordgren, J. Electron Spectrosc. Relat. Phenom. 82, 193 (1996).

[28] J. A. Fleck, Phys. Rev. B 1, 84 (1970).

[29] N. Kosugi and H. Kuroda, Chem. Phys. Lett. 74, 490 (1980); N. Kosugi, Theor. Chim. Acta 72, 149 (1987).

[30] O. Larroche, D. Ros, A. Klisnick, A. Sureau, C. Möller, and H. Guennou, Phys. Rev. A 62, 043815 (2000).

[31] L. Casperson and A. Yariv, Phys. Rev. Lett. 26, 293 (1971).

[32] M. Shapiro, J. Chem. Phys. 99, 2453 (1993).

[33] F. Gel'mukhanov, P. Salek, A. Shalagin, and H. Agren, J. Chem. Phys. 112, 5593 (2000).

[34] F. Gelmukhanov and H. Ågren, Phys. Rep. 312, 87 (1999).

[35] F.F. Guimarães, V. Kimberg, V.C. Felicissimo, F. Gel'mukhanov, A. Cesar, and H. Ågren, Phys. Rev. A 71, 043407 (2005). 